\title{
Espacio fiscal para el financiamiento sostenible de los sistemas de salud y la salud universal
}

\author{
Camilo Cid Pedraza, ${ }^{1}$ Juan Pablo Pagano, ${ }^{1}$ Claudia Pescetto ${ }^{1}$ \\ y Lorena Prieto ${ }^{1}$
}

Forma de citar

Cid Pedraza C, Pagano JP, Pescetto C, Prieto L. Espacio fiscal para el financiamiento sostenible de los sistemas de salud y la salud universal. Rev Panam Salud Publica. 2018;42:e197. https://doi.org/10.26633/ RPSP.2018.197

RESUMEN Los artículos publicados en esta serie muestran que es posible crear espacio fiscal para la salud en los países. Para esto se requieren decisiones específicas, ya que el crecimiento económico no es suficiente para generar los recursos adicionales necesarios. Los estudios analizan la conveniencia de revisar los gastos tributarios para identificar las exenciones -en general desactualizadas y de escasos beneficios para los países-; los argumentos para aumentar los impuestos sobre productos dañinos para la salud; y los créditos y donaciones, que no resultan una fuente de ingresos viable para los gobiernos. Los esfuerzos fiscales deben ser acompañados por una mejora de la eficiencia, y la creación progresiva de nuevos ingresos es clave para mejorar la equidad. Es necesario mejorar la recaudación de los recursos fiscales.

Para esto se requiere desarrollar una agenda de investigación y acción que entienda el análisis del espacio fiscal inserto en los procesos de transformación y reforma de los sistemas de salud, que abarque los aspectos técnicos no abordados aún y estudios de la eficiencia social del espacio fiscal para los grandes objetivos de desarrollo como los Objetivos de Desarrollo Sostenible 2030. En los países de la Región de las Américas es imprescindible contar con una gestión eficiente para hacer más y mejor con más recursos, incluso durante los ciclos económicos adversos. Esta debe evidenciarse en todos los niveles, incluidas la solidaridad que logra mejores resultados en salud, los sistemas de asignación de recursos a los proveedores, la compra estratégica de bienes y servicios de salud y el fortalecimiento de los sistemas de planificación presupuestaria.

Palabras clave

Financiamiento de la salud; sistema de salud; impuestos; equidad en salud; eficiencia Américas.

En esta serie se ha presentado un conjunto de artículos sobre financiamiento y espacio fiscal para la salud en los países de las Américas. La serie comienza con

\footnotetext{
Departamento de Sistemas y Servicios de Salud. Organización Panamericana de la Salud, Washington D.C., Estados Unidos de América. Enviar correspondencia a Camilo Cid Pedraza, cidcam@paho.org
}

dos artículos: un editorial de la directora de la OPS—que presenta el marco de política que fundamenta la necesidad del aumento del gasto público en salud y la disminución del pago directo de bolsillo (1) - y un análisis regional sobre la posibilidad de ese aumento, que cuenta solo con condiciones macroeconómicas propicias traducidas en crecimiento y concluye que no es suficiente, que el inmovilismo resulta improductivo y que es necesario tomar medidas específicas para aumentar el espacio fiscal para salud en los países si se quiere avanzar hacia la salud universal (2).

Los trabajos posteriores sobre espacio fiscal para Perú (3), Bolivia (4), Honduras (5) y Suriname (6) avanzan en líneas específicas con una metodología común que se compone de aspectos técnicos y

Este es un artículo de acceso abierto distribuido bajo los términos de la licencia Creative Commons Attribution-NonCommercial-NoDerivs 3.0 IGO, que permite su uso, distribución y reproducción en cualquier medio, siempre que el trabajo original se cite de la manera adecuada. No se permiten modificaciones a los artículos ni su uso comercial. Al reproducir un artículo no debe haber ningún indicio de que la OPS o el artículo avalan a una organización o un producto específico. El uso del logo de la OPS no está permitido. Esta leyenda debe conservarse, junto con la URL original del artículo. 
considera cada una de las fuentes de espacio fiscal en el caso concreto de cada país, y de economía política.

El artículo sobre cuentas de salud entabla una relación entre la contabilidad del financiamiento como medio para conocer y monitorizar la inversión en salud y las políticas de aumento del espacio fiscal (7). El trabajo de gasto de bolsillo en Perú muestra los determinantes en los más pobres y señala la importancia de reorientar las políticas de protección financiera con mayor gasto público (8). El estudio de Granada indaga en el impuesto específico al tabaco como posible fuente de financiamiento (9).

En este artículo de cierre de la serie se destacan los principales aspectos de los trabajos publicados y se releva la importancia de presentar una direccionalidad para los avances futuros en este campo. Se destaca que estos se concretan no solo en la elaboración de nuevos estudios, sino también en la interacción entre distintas actividades en los países que releven sus necesidades de financiamiento para la sostenibilidad y los cambios necesarios de los sistemas de salud.

\section{MATERIALES Y MÉTODOS}

El surgimiento del concepto de espacio fiscal y su vínculo con la salud ocurre a propósito de los esfuerzos por cumplir los Objetivos de Desarrollo del Milenio, fue sistematizado por funcionarios del Fondo Monetario Internacional y el Banco Mundial, sobre todo durante la década del 2000 (10) y fue reconsiderado por la Organización Panamericana de la Salud/Organización Mundial de la Salud (OPS/OMS) en el contexto del impulso de la Estrategia de Salud Universal en 2014 y el compromiso subsecuente por aumentar con eficiencia y equidad el gasto público en salud para eliminar, en forma paulatina, el gasto de bolsillo (11).

El concepto hace referencia a la capacidad de los gobiernos de asignar recursos presupuestarios adicionales para el sistema de salud, sin afectar la situación financiera del sector público ni desplazar otros gastos sociales necesarios (12). Destaca, también, la condición de sustentabilidad de la posición financiera de los gobiernos en procesos en los que se necesita contar con recursos adicionales a los existentes para destinarlos a la salud, en este caso, para avanzar hacia el acceso y la cobertura universal.
Las fuentes de espacio fiscal que se han identificado son: a) crecimiento económico y creación de las condiciones macroeconómicas propicias; b) una mayor priorización para la salud; c) creación de nuevos ingresos fiscales y mayor presión fiscal; d) ayuda externa en forma de préstamos y donaciones para el sector de la salud y e) aumento en la eficiencia de los gastos de salud existentes (10).

El método desarrollado por los estudios de espacio fiscal consiste en un diagnóstico sobre las potenciales fuentes de recursos adicionales para el sistema de salud y su evaluación en términos de cuantía y viabilidad política en cada caso concreto. En este contexto, se busca dilucidar la forma de obtener más ingresos vía impuestos u otros medios, considerando que la distribución del gasto, las mejoras organizacionales que conduzcan a una mayor equidad y los procesos de aceptación política de las medidas tienen, todos, el mismo grado de importancia.

En los estudios complementarios los métodos son variados y cumplen con el

\section{FIGURA 1. Las cinco fuentes de espacio fiscal para la salud}

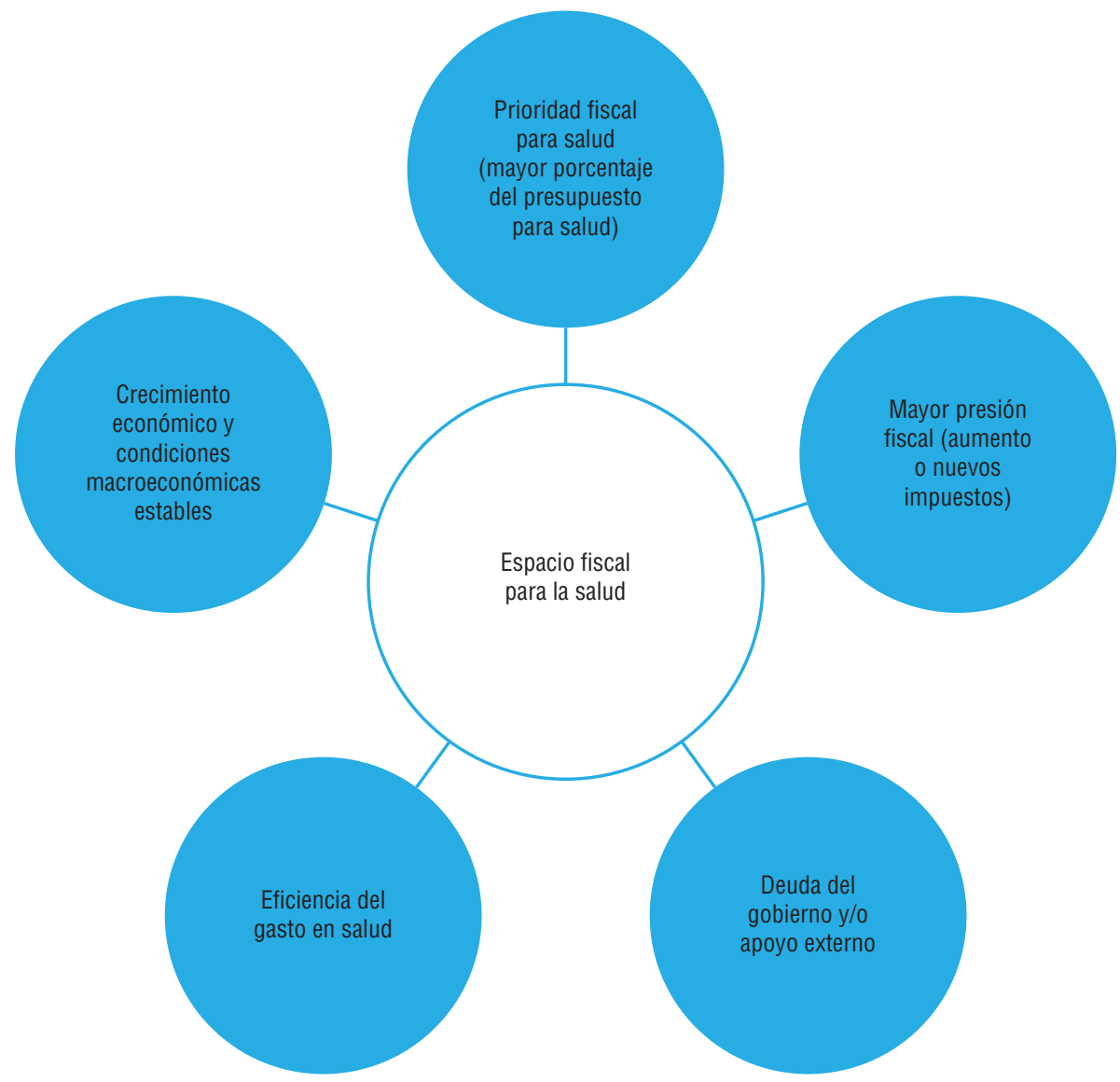

Fuente: elaboración propia. objeto de sustentar las hipótesis tratadas: un método descriptivo en el tema de cuentas de salud, indicadores, su utilidad e historia; uno econométrico para establecer determinantes e impactos de los gastos de bolsillo, y uno específico de medición de cuantía de un alza a impuestos al tabaco.

En el cuadro 1 se muestran las temáticas tratadas por los seis artículos que se refieren a medición de espacio fiscal.

\section{RESULTADOS Y DISCUSIÓN}

El crecimiento económico y las condiciones macroeconómicas propicias son la fuente más estudiada de espacio fiscal. En general, en una economía que crece de manera estable, al aumentar la recaudación fiscal y, de mantenerse la distribución del gasto público por sectores del gobierno, sería esperable un aumento de los recursos económicos para la salud, pero se ha mostrado que esto no es suficiente.

De hecho, si se mantuviera la forma de priorizar salud de los últimos 20 años 
CUADRO 1. Fuentes de espacio fiscal tratadas por los autores de los artículos de la seriea

\begin{tabular}{|c|c|c|c|c|c|c|}
\hline \multirow{2}{*}{ Fuente de espacio fiscal } & \multicolumn{6}{|c|}{ Estudio (referencia) } \\
\hline & Regional (2) & Bolivia (4) & Granada (9) & Honduras (5) & Perú (3) & Suriname (6) \\
\hline Crecimiento económico/condiciones macroeconómicas propicias & $\bullet$ & $\bullet$ & & $\bullet$ & $\bullet$ & $\bullet$ \\
\hline Repriorización del gasto en salud & & $\bullet$ & & $\bullet$ & $\bullet$ & $\bullet$ \\
\hline Nuevos ingresos tributarios y contribuciones sociales para salud & & $\bullet$ & & $\bullet$ & $\bullet$ & $\bullet$ \\
\hline Aumento de impuestos & & $\bullet$ & & • & $\bullet$ & $\bullet$ \\
\hline Impuestos generales & & $\bullet$ & & & & \\
\hline Impuestos directos & & & & $\bullet$ & & \\
\hline Impuestos indirectos & & & & $\bullet$ & & \\
\hline Impuestos específicos de salud públicab & & $\bullet$ & $\bullet$ & $\bullet$ & $\bullet$ & $\bullet$ \\
\hline Tabaco & & & $\bullet$ & & & \\
\hline Contribuciones sociales en salud & & $\bullet$ & & $\bullet$ & $\bullet$ & $\bullet$ \\
\hline Reducción de los gastos tributarios & & $\bullet$ & & $\bullet$ & $\bullet$ & $\bullet$ \\
\hline Impuestos sobre recursos naturales & & $\bullet$ & & & & \\
\hline Ayuda externa y obtención de préstamos & & $\bullet$ & & $\bullet$ & $\bullet$ & \\
\hline Mejoras en eficiencia & & $\bullet$ & & $\bullet$ & $\bullet$ & $\bullet$ \\
\hline
\end{tabular}

a Solo se incluyen los seis artículos que miden espacio fiscal.

${ }^{b}$ Impuestos a productos dañinos y marcados.

Fuente: elaboración propia. Adaptado de OPS, 2018 (10).

en la Región, con base en el crecimiento económico futuro, excepto los tres países de América Latina y el Caribe que ya alcanzaron la meta, solo cuatro países más lograrían alcanzar $6 \%$ del producto interno bruto (PIB) de gasto público en salud antes de 2030 y muchos lo harían hacia fin de siglo o nunca (2).

Dada la baja prioridad fiscal para la salud en la Región $(10,13)$, si bien la repriorización del presupuesto de salud dentro del gasto público total aparece como una fuente importante de espacio fiscal, se encuentra con la dificultad de la estructura rígida del gasto público.

Existen dos consideraciones para abordar la repriorización: como un incremento del peso del gasto en salud en relación con el gasto público total, o bien como un aumento en el conjunto del gasto social. En este último caso, se busca evitar una competencia del gasto en salud con otras partidas que son complementarias en un abordaje intersectorial de la salud, y se apunta entonces a una repriorización en el margen del aumento presupuestario.

Así, por ejemplo, en el caso de Perú, el problema de financiamiento del sector salud se relaciona más con la dimensión del total de los ingresos públicos que con la priorización en salud (3). En Bolivia, sin embargo, se observa una recaudación pública moderadamente alta, pero con una baja prioridad fiscal para la salud, lo que da lugar a ganancias potenciales de espacio fiscal por esta vía (4).
La fuente de nuevos ingresos se divide en ingresos tributarios (impuestos) y contribuciones sociales obligatorias. Los impuestos son la fuente que presenta más oportunidades, que van desde el aumento de nivel de la carga tributaria y la creación de nuevos impuestos (incluidos los que gravan a bienes dañinos para la salud), hasta la reducción del gasto tributario o exenciones. Lo que se busca es aumentar la recaudación y, a la vez, dedicar parte de ese aumento a salud. Se trata de un esfuerzo activo y no solo inercial.

Al momento de estas decisiones políticas, se debe tener en cuenta que los sistemas tributarios basados en su mayoría en impuestos indirectos, como la mayoría de los países de la Región, tienden a ser regresivos. Los impuestos progresivos (como los que gravan la renta y el patrimonio) son deseables, tienen un impacto en la equidad general y la evidencia muestra que están asociados también con mejoras en la situación de salud $(14,15)$. También se ha de tener presente la baja presión fiscal de los países de la Región que, siendo un problema, se presenta a la vez como una oportunidad.

En este contexto, Perú ha favorecido la ampliación de la carga tributaria progresiva relacionada con el ingreso, pero sin excluir la posibilidad de aumentar la tributación asociada a la explotación de los recursos naturales (3). En Bolivia parece existir margen para el aumento de la recaudación a través de impuestos directos, y menos por impuestos que gravan el consumo. Reducir una cuarta parte la brecha impositiva sobre renta y beneficios con respecto a los países de la Región, podría liberar casi medio punto del PIB en este país.

En cuanto al aumento de los impuestos específicos sobre bienes dañinos para la salud, ello parece factible en el caso del impuesto al tabaco en Bolivia y Perú, pero con un impacto reducido en términos de monto. No obstante, esta es una medida de salud pública deseable y existen posibles ahorros futuros para la sociedad derivados de una reducción en la utilización de los servicios de salud de alto costo y una disminución de la mortalidad prematura en estos casos.

En 2013, se implementó en Perú un aumento del impuesto selectivo al consumo sobre cervezas, vinos y licores y, en 2016, en paralelo a la realización del estudio de espacio fiscal, se incrementó el impuesto selectivo al consumo (ISC) de los productos derivados del tabaco. El ISC volvió a aumentar en 2018, sin que se destinara de manera específica a salud. Por lo tanto, en la actualidad, resulta poco probable que se den otros aumentos. Por su parte, la última reforma tributaria en Honduras tuvo la misma implicancia al aumentar el impuesto a los alcoholes $(4,5)$.

Los gastos tributarios o "renuncias fiscales" (reducciones de ingresos fiscales relacionadas con exenciones, reintegros, deducciones, etc.) son una de las fuentes más importantes de espacio fiscal. 
El grueso de ellos, en Honduras, representa casi la misma proporción que los impuestos sobre las ventas y de importación, esto es, cerca de $30 \%$ del total de la recaudación (5). Una decisión política para reducirlos podría aumentar la recaudación en más de un punto del PIB en ese país. En Perú se estima un espacio fiscal de casi medio punto del PIB ante una medida similar.

En Suriname, el efecto del subsidio a la electricidad es inusualmente alto con varios puntos del PIB (6). El reto en esta fuente está en lograr la decisión política de redireccionarla, sobre todo frente a los sectores específicos que podrían verse afectados. En cualquier caso, se debe tener en cuenta que muchas de estas excepciones se crearon en momentos específicos $\mathrm{y}$ con fines concretos, pero no se han vuelto a evaluar.

El efecto de una reducción de la economía informal es otra de las fuentes de espacio fiscal consideradas. Este es un asunto complejo que involucra al mercado laboral informal, que tienen su propia dinámica y factores determinantes. El gran reto, en este caso, es la generación de políticas que logren la reducción de la informalidad y el aumento de la capacidad de recaudación, tanto por contribuciones como por ingresos tributarios, lo que implica una acción del conjunto del gobierno.

En tanto fuente, surge la disyuntiva sobre la segmentación generada por las condiciones del mercado laboral versus priorizar fuentes que no la fomente, como es el caso de los ingresos tributarios y que beneficien no solo a los trabajadores formalizados.

Los estudios presentan estimaciones que en algunos casos dan cuenta de niveles iniciales muy bajos de contribuciones (como en Honduras) y, por tanto, de una fuente de gran potencial, aunque mas por la vía de extender la cotización y sus techos de cálculo, que por la vía de extender la base de trabajadores formales (5).

Por otra parte, en el campo de los impuestos aparecen la necesidad de aumento en la eficiencia de la recaudación que hace referencia a evitar la evasión y la elusión tributaria y también fomentar la formalidad del mercado del trabajo; por supuesto, como se ha señalado, se trata de acciones que abarcan a los gobiernos en su conjunto.

Respecto de las fuentes externas de financiamiento, si bien existe cierta aceptación cuando se trata de inversión en infraestructura, los estudios no suelen recomendarlas como una fuente deseable de espacio fiscal debido a su volatilidad, falta de sostenibilidad y a la fragmentación que provocan, en el sistema de salud, las formas de implementación de este tipo de financiamiento.

Incluso, en Honduras se asocian con un intercambio entre ayuda externa y recursos propios, y la priorización de otros sectores, a los que se desplaza la asignación de fondos de impuestos generales a raíz del aumento de la ayuda externa para el sector de la salud. Los nuevos recursos no han contribuido a aumentar el presupuesto, sino a liberar los existentes hacia otros sectores (5). A ello habría que agregar su efecto en segmentación, ya que su operación, en general vertical, da lugar a una actividad estanca en todas las fases del financiamiento.

La eficiencia es una fuente particular de espacio fiscal y la más atractiva en términos políticos. No obstante, en los estudios analizados, la cuantificación del nivel de eficiencia del gasto en salud es compleja. Al considerar los rendimientos marginales decrecientes del gasto, se ha estimado que ganancias relativamente menores de eficiencia podrían generar un espacio fiscal relevante.

Se destacan la potencialidad del mejoramiento de los mecanismos de pago y distribución del gasto público en salud $\mathrm{y}$, en particular, de reducir las ineficiencias e inequidades que representan los seguros de salud establecidos como compartimentos estancos y sin canales de solidaridad $(3,4)$.

La segmentación en el aseguramiento desaprovecha la eficiencia de las economías de escala y las ganancias de la distribución del riesgo en la mancomunación, a la vez que incrementa las asimetrías de información y favorece los procesos de selección adversa de la población beneficiaria según el riesgo de enfermar.

Respecto de estos dos últimos puntos, los sistemas de pago y la mancomunación, el caso de Suriname es en particular ilustrativo, pues cuenta con la oportunidad de extender su fondo nacional de salud (SZF, por sus siglas en holandés) en función de aplicar mejores formas de pagos y aprovechar así las ganancias de eficiencia (6).

Por último, en el análisis de viabilidad político-social de intervenciones planteadas en los trabajos, es posible identificar rasgos comunes, como por ejemplo la preferencia por parte de los actores consultados, por la ampliación de la base impositiva y la disposición generalizada a aumentar los impuestos a bienes dañinos para la salud.

El aumento de la eficiencia mantiene un predecible consenso, mientras que la propuesta de aumentar la presión fiscal con reformas tributarias progresivas tiene una recepción más dividida, pero sin excluir sus posibilidades. En el cuadro 2 se resumen los principales de los hallazgos descritos.

Por último, los estudios han destacado que el espacio fiscal para la salud se centra en la capacidad y viabilidad de fuentes adicionales de financiamiento, pero no responde a todos los interrogantes $\mathrm{y}$ aspectos relacionados con el gasto en salud.

No obstante, la vinculación con el camino de transformación del sistema de salud es crucial, así como responder de manera efectiva si las intervenciones podrán ser financiadas de manera sostenible.

El volumen de recursos necesarios para avanzar hacia la salud universal depende de los costos de la atención, la infraestructura, el perfil epidemiológico, la carga de enfermedad en cada país y los precios nacionales $y$, por lo tanto, pueden implicar esfuerzos y retos diferentes en cada país.

Es por esto que la pregunta acerca de los próximos pasos debe asociarse a una agenda de investigación y acción que se desprende de los estudios dirigidas a asociar más los recursos con los objetivos de salud universal, de modo de conocer los costos de ese camino de reforma y transformación, en función de los requerimientos de cada país.

\section{Una agenda para la investigación y para la acción}

Numerosos estudios muestran los beneficios de invertir en salud en tanto inversión en capital humano (16-18): se rescata a las personas de la pobreza derivada de tener que pagar por servicios médicos en el punto de servicio, se incrementa la productividad y los niveles de ingreso, se fomenta el crecimiento económico (19-29) y, sobre todo, se mejora la salud y el bienestar de la población (30-32).

El sector de salud también es considerado como uno de los sectores más efectivos donde invertir para disminuir inequidades, junto con educación; de esta 
CUADRO 2. Resumen de hallazgos de los estudios publicados en esta serie

\begin{tabular}{|c|c|c|}
\hline Fuente de espacio fiscal & Factibilidad técnica & Factibilidad política \\
\hline $\begin{array}{l}\text { Crecimiento económico y condiciones } \\
\text { económicas propicias }\end{array}$ & $\begin{array}{l}\text { Es la fuente inercial; sin embargo, evaluada de acuerdo a períodos de } \\
\text { crecimiento mayores que los actuales y futuros, no ha sido suficiente }\end{array}$ & $\begin{array}{l}\text { Aumentar el gasto público en salud por lo menos } \\
\text { al mismo ritmo del crecimiento económico tiene } \\
\text { aceptación casi universal }\end{array}$ \\
\hline $\begin{array}{l}\text { Repriorización del presupuesto } \\
\text { para salud }\end{array}$ & $\begin{array}{l}\text { El simple aumento del presupuesto es factible por cuanto el peso de los } \\
\text { presupuestos para salud es bajo. Desde un punto de vista técnico, ha de } \\
\text { considerarse la relación sectorial con los determinantes de la salud, y que } \\
\text { la diminución de sectores asociados a salud pueda ser contraproducente. }\end{array}$ & $\begin{array}{l}\text { Existe importante rigidez del presupuesto público } \\
\text { entre sectores }\end{array}$ \\
\hline \multicolumn{3}{|l|}{ Nuevos ingresos } \\
\hline Impuestos generales & $\begin{array}{l}\text { El IVA suele ser un recurso de uso rápido y fácil, pero regresivo } \\
\text { Impuestos a la renta suelen requerir una reforma fiscal }\end{array}$ & $\begin{array}{l}\text { El IVA es complejo por su regresividad } \\
\text { Impuesto a la renta dificultoso por la capacidad de } \\
\text { lobby y oposición de los afectados }\end{array}$ \\
\hline $\begin{array}{l}\text { Impuestos específicos e impuestos } \\
\text { marcados }\end{array}$ & $\begin{array}{l}\text { Impuestos al tabaco, alcoholes, bebidas azucaradas y alimentos } \\
\text { procesados, son de fácil cálculo y operación }\end{array}$ & $\begin{array}{l}\text { Muy factibles y de fácil comprensión del público, } \\
\text { pero dadas las reformas recientes en algunos países } \\
\text { no es posible }\end{array}$ \\
\hline Gastos fiscales & Bastante fáciles de identificar, según el contexto & $\begin{array}{l}\text { Requiere decisión política y diálogo posible entre los } \\
\text { actores, ya que habrá resistencia de los afectados }\end{array}$ \\
\hline $\begin{array}{l}\text { Contribuciones de seguridad social } \\
\text { de salud (formalización) }\end{array}$ & $\begin{array}{l}\text { De eficacia restringida; dada la informalidad del mercado laboral } \\
\text { y la segmentación de los sistemas, suele beneficiar solo a los } \\
\text { trabajadores formales. }\end{array}$ & $\begin{array}{l}\text { Depende de variables asociadas a la economía y } \\
\text { políticas para mejorar el mercado laboral }\end{array}$ \\
\hline Ayuda externa & $\begin{array}{l}\text { Casi no quedan países que clasifiquen en las ayudas más básicas. } \\
\text { Los préstamos son sencillos, pero suelen acarrear problemas de } \\
\text { segmentación y verticalidad programática }\end{array}$ & $\begin{array}{l}\text { No es considerada como posibilidad, dada la } \\
\text { volatilidad y sostenibilidad en el largo plazo }\end{array}$ \\
\hline Eficiencia & $\begin{array}{l}\text { De la mano de los procesos de transformación es posible identificar } \\
\text { acciones para la eficiencia, como el cambio hacia la operación en RISS } \\
\text { con APS resolutiva y aprovechar la eficiencia del fondo de mancomunación } \\
\text { amplio que tienda a ser único y así mitigar la segmentación }\end{array}$ & $\begin{array}{l}\text { Tiene aceptación universal. Sin embargo, al momento } \\
\text { de aplicar medidas, se puede presentar resistencia si } \\
\text { no hay acuerdos previos entre los actores del sector }\end{array}$ \\
\hline
\end{tabular}

IVA, impuesto al valor agregado; RISS, redes integradas de servicios de salud; APS, atención primaria de salud.

Fuente: elaboración propia.

forma se relaciona el incremento en espacio fiscal también para la reducción de la inequidad $(33,34)$.

Se requiere el desarrollo de una agenda de investigación para la toma de decisiones que extienda el análisis de espacio fiscal, tanto hacia aspectos técnicos no abordados hasta ahora, como a áreas de abogacía más explícita asociada a los costos de las transformaciones necesarias de los sistemas de salud.

Como se mencionó, resulta necesario conocer la magnitud del esfuerzo por realizar en cada caso. En este sentido, es siempre oportuno dimensionar el costo de los cambios y de las brechas materiales y de recursos humanos que implican, para determinar en forma exacta cuántos recursos extra se precisan para avanzar.

De este modo, los países que avanzan en salud y salen de un estancamiento previo asociado al dominio de la segmentación y fragmentación y eligen el camino de la salud universal, enfrentan decisiones en ambos sentidos: en los métodos de cuantificación y el análisis de viabilidad política $(35,36)$.

Lo anterior tiene implicancias en cada una de las fases del financiamiento de la salud y del sistema de salud, las fuentes, la mancomunación de recursos y la asignación de estos a los proveedores. En la figura 2 se resumen estos desafíos que detallamos a continuación, con la eficiencia como un elemento transversal.

En cuanto a las fuentes de financiamiento, es relevante la discusión sobre el desarrollo de herramientas concretas para aumentar el espacio fiscal y proteger el gasto público en salud de eventualidades del ciclo económico y político. Por ejemplo, la discusión acerca de la prioridad fiscal para salud y de las ventajas y desventajas de los impuestos marcados (earmarking, asignación de recursos públicos específicos para un fin específico) debería brindar elementos importantes para la toma de decisiones en la Región.

En este sentido, se puede analizar dicha herramienta reconociendo al menos dos tipologías: earmarking de fuentes de recursos (asegurar los recursos provenientes de fuente pública para un fin específico) y de asignación o gasto (una proporción determinada del gasto público general asignada a un destino) (37).

Además, la noción de eficiencia en salud como fuente de espacio fiscal requiere nuevos desarrollos, tanto analíticos como conceptuales, ya que si bien, en sentido estricto, liberar recursos dentro del mismo sector no genera recursos adicionales para este, sino que una reasignación resultante de ganancias en eficiencia permitiría expandir el gasto en otros programas, dentro del sector salud mismo.

Por ejemplo, si se mejora la eficiencia de las compras públicas de medicamentos, con acceso a mejores precios y reducción del gasto en ese rubro específico, se podrían utilizar los recursos provenientes de dicho ahorro para la contratación de un número mayor de recursos humanos en salud en áreas desatendidas.

En cualquier caso, una agenda de investigación de eficiencia en salud debería construirse sobre la base de al menos dos premisas: no se trata de una dicotomía entre aumentar los recursos a salud o utilizarlos en forma más eficiente, sino más bien una combinación de ambos enfoques; por otra parte, no se trata de cómo recortar los presupuestos públicos (hacer lo mismo con menos recursos, un objetivo no deseable en salud). En este sentido, la eficiencia en salud aparece como un elemento que es transversal a las funciones básicas de financiamiento en salud (38).

Un nuevo desarrollo implica incorporar el análisis de opciones de optimización de la eficiencia social en la 
FIGURA 2. Agenda para investigación y acción en espacio fiscal para salud universal
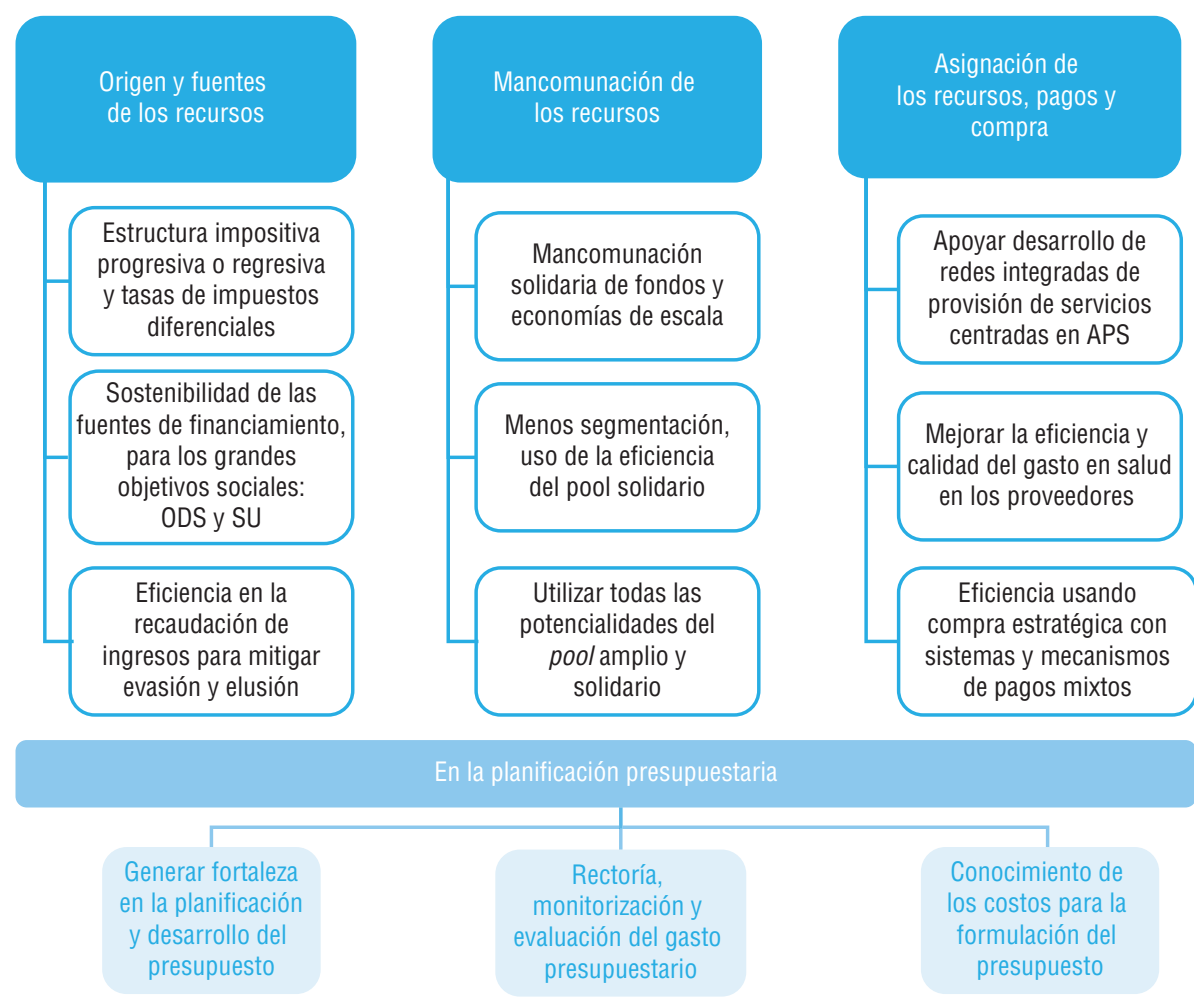

APS, atención primaria de salud; ODS; Objetivos de Desarrollo Sostenible; SU, salud universal. Fuente: elaboración propia.

consecución de los grandes objetivos de desarrollo, como son la salud universal y los Objetivos de Desarrollo Sostenible (ODS) 2030. Estos incluyen, de manera inevitable, la discusión del conjunto de los sectores sociales y económicos de los Estados sobre los sistemas impositivos y su capacidad de dar cuenta de estos objetivos de desarrollo humano, haciéndolos parte de las evaluaciones globales de desempeño en cuanto a eficiencia y equidad del gasto público.

Deberían ser reforzados algunos asuntos aún no tratados, como son el aporte potencial de los impuestos subnacionales o la temática de la eficiencia en la recaudación de ingresos fiscales y la promoción de la progresividad y de aumento de la presión fiscal del sistema tributario.

Por otra parte, debido a razones técnicas y políticas, los países presentan tasas impositivas efectivas diferenciadas por sector económico. Sin embargo, aunque mejorar dichas inequidades -muchas veces poco justificadas o simplemente inerciales- no se traduzca siempre en un aumento de recursos para salud, la presencia de estudios de espacio fiscal que visibilicen estos aspectos podría incentivar un diálogo social en los países y dar soporte a favor de la inversión en salud.

Además, cualquier estudio de fuentes de recursos para ampliar el espacio fiscal para salud deberá contener un fuerte componente de economía política y viabilidad de cada una de las fuentes potenciales.

Con respecto a las fuentes externas de recursos, en especial aquellas referentes a donaciones y ayuda internacional, se debe discutir el tema de la eficiencia en la provisión de aquellos programas prioritarios de salud que hoy en día se benefician de dicha cooperación.

El enfoque de esos casos debería ser el de sostenibilidad e integración de la respuesta del sistema de salud a dichos programas y no tanto de sostenibilidad de acciones específicas en salud aisladas del resto del sistema. Es decir, cómo avanzar en modelos más eficientes de provisión de dichos servicios, en preparación para una transición hacia un financiamiento doméstico, en vistas de que la mayoría de los países de la Región -en forma lenta pero sostenida- comienzan a dejar de ser elegibles para esa ayuda por motivos de crecimiento económico y de disminución de la carga de enfermedad (39). En este sentido, es necesaria una perspectiva de integración y elementos concretos para avanzar en dicha dirección.

Se podrá estudiar la costo-efectividad de las acciones, que no es sino estimar la eficiencia técnica de algunas intervenciones. Esto puede guiar la toma de decisiones respecto de qué intervenciones deben continuar y cuáles deben ser reformuladas en una perspectiva de financiamiento doméstico.

Respecto de la mancomunación, los países se beneficiarían de análisis específicos que cuantifiquen las ganancias de eficiencias provenientes de mejorar dicha función. Más allá de los posibles ahorros en gastos administrativos de reducir el número de fondos y de aumentar el tamaño de la mancomunación, se requiere analizar cómo la función misma de aseguramiento se mejora cuando el tamaño del grupo de personas protegidas aumenta, debido a economías de escala y a una mejora en la distribución de los riesgos.

Este último aspecto, además de ser relevante para una Región caracterizada por una alta segmentación de sus sistemas de salud, es de especial relevancia para los países de la subregión del Caribe, que cuentan con poblaciones relativamente más pequeñas.

Además de la segmentación (entendida como esquemas específicos que protegen segmentos específicos de la población según ocupación o nivel socio-económico), en muchos casos la función de mancomunación puede estar fragmentada por otros motivos como la regionalización y descentralización de algunos sistemas de salud de la Región, que deberán analizarse con un lente de eficiencia también.

En el mismo sentido, la organización y financiamiento vertical de algunos programas de salud pública también genera obstáculos al mejoramiento de la función de mancomunación y el análisis de casos específicos y opciones concretas sobre cómo avanzar también aportaría elementos importantes para la toma de decisiones en algunos países de la Región.

Una perspectiva poco explorada, y necesaria, en la Región es identificar evidencia que muestra que esto tiene 
implicancias en la salud; por ejemplo, una mancomunación solidaria amplia o un aumento de esta, se asocia a mejores resultados de salud, como consta en la literatura (40).

En el ámbito de la asignación de recursos y sistemas de pago para servicios de salud, el análisis de las posibles fuentes de ineficiencias que se desprenden de los mecanismos de asignación preponderantes en los países, así como de la administración de las finanzas públicas y el presupuesto en general, son temáticas vigentes.

La predominancia de la asignación de recursos hacia los prestadores públicos mediante presupuestos históricos influidos por la presión (lobby) y con poca relación con los costos poco aportan en incentivar la eficiencia y en abordar los incentivos débiles a la productividad en el sector público (38).

Además, se podrán visibilizar los ahorros potenciales por la posibilidad acceder a mecanismos de compra de medicamentos conjunta, regionales o subregionales, como el Fondo Estratégico de la Organización Panamericana de la Salud (OPS) o el esquema de compras farmacéuticas de la Organización de Estados del Caribe del Este (OECS).

Al relacionar la eficiencia y la organización del sistema de atención de salud, se puede explorar en una discusión retrospectiva que muestre cómo lograron ampliar el espacio fiscal para salud aquellos países que más avanzaron en dicha materia en la Región, como Costa Rica, Cuba o Uruguay. Es importante no dejar de reconocer la necesidad de procesos que avancen en la dirección de asegurar una atención integral (y a través de redes integradas de servicios de salud) para todos y para esto brindar instrumentos técnicos que faciliten su implementación de manera eficiente y realista.

Los países precisan avanzar hacia un horizonte definido que aborde los problemas estructurales de los sistemas. Estas transformaciones deben apuntar a la eficiencia del gasto con equidad, apuntar a la mancomunación solidaria única, crear o fortalecer sistemas integrales e integrados de salud, con un primer nivel de atención resolutivo y articulador de redes y basado en un enfoque de atención primaria de salud que brinda no solo servicios curativos, sino también de promoción y prevención en salud, en función de que se sabe que este aumento y su perspectiva puede mejorar la salud de la población como lo demuestra la evidencia $(41,42)$.

Los sistemas de asignación de recursos, mecanismos de pago y la compra estratégica son un campo de estudio en sí mismos, deben reconocerse como elementos necesarios para la mejora de la eficiencia en un contexto de necesidad de espacio fiscal y deben perseguir el objetivo de apoyar los esfuerzos de mejoramiento del sistema integrado que se reseña.

A su vez, la posible disponibilidad de espacio fiscal debe priorizar la implementación de estos enfoques, que se utilicen todos los recursos disponibles en la sociedad y se evite el pago en el punto de servicio. Se trata de entender la necesidad de aumentar el espacio fiscal para la salud con la eficiencia como una condición necesaria, enmarcada en un proyecto de transformación de los sistemas de salud.

En ese contexto, con el sector público en un rol fundamental, la presupuestación en todas sus fases (planificación, ejecución, monitorización) como parte relevante de los mecanismos de planificación en salud es un pilar de la sostenibilidad y eficiencia del sistema. Este pilar debe ser fortalecido y arraigado en los costos reales, incluso a veces construido como primera prioridad en el ámbito del financiamiento y forma de pagos dada la debilidad del sistema de financiamiento $y$, otras veces, reconstruido después de intervenciones desafortunadas.

Por último, no es posible obviar el contexto macroeconómico de crisis o de crecimiento lento en algunos países, en particular en América del Sur, en los últimos años.

En 2018, el crecimiento medio en América Latina y el Caribe será de 1,5\% (43) ${ }^{2}$.

\footnotetext{
2 La Comisión Económica para América Latina y el Caribe (CEPAL) estima que las economías de América Latina y el Caribe crecerán gracias a un repunte de la demanda interna. Se espera que América del Sur crezca 1,2\% en 2018, mientras que América Central lo haría en 3,4\% y el Caribe en 1,7\%. República Dominicana y Panamá liderarán el crecimiento de la región, con aumentos del producto interno bruto (PIB) de 5,4\% y 5,2\%, respectivamente, seguidos por Paraguay $(4,4 \%)$, Bolivia (4,3\%), Antigua y Barbuda (4,2\%). Este crecimiento regional se da en un escenario global complejo, caracterizado por conflictos comerciales entre Estados Unidos, China y otras naciones,
}

En este contexto, una agenda de promoción de inversión en salud necesita apelar a la política contracíclica para moderar el ciclo en vez de acompañarlo con medidas como el alza de los tipos de interés y reducción presupuestaria y del gasto social, que suelen profundizar la caída del producto (44).

Las lecciones aprendidas apuntan más bien a apoyar la demanda interna, reforzar los resultados fiscales primarios (previos al pago de deuda externa) que en la Región están en franca mejoría y respaldar las medidas de reactivación de la política fiscal (43), más que promover la austeridad de recortes presupuestarios lineales que ponen en peligro la sostenibilidad de los sistemas de salud, como lo ha mostrado parte de la experiencia global $(45,46)$.

El espacio ofrecido por los problemas estructurales de segmentación y fragmentación de la gran mayoría de los sistemas de salud en la Región (y de los países con mayores problemas económicos), incluso en contexto de complicaciones económicas, son también una oportunidad de acompasar la transformación, eficiencia y sostenibilidad de logros alcanzados, en un esquema virtuoso de desarrollo de políticas fiscales, sociales y de salud.

Así lo entendieron algunos países, como se puede apreciar en algunos casos del sudeste asiático, que montaron importantes reformas sociales y de salud como parte importante de las medidas de salida de la crisis de 1997-98 en esa subregión (47).

Conflicto de intereses. Ninguno declarado por los autores.

Declaración. Las opiniones expresadas en este manuscrito son responsabilidad del autor y no reflejan necesariamente los criterios ni la política de la RPSP/ $P A J P H$ y/o de la OPS.

\footnotetext{
riesgos geopolíticos crecientes y una expansión económica mundial que tiende a perder dinamismo. La recaudación tributaria de América Latina se mantiene estable, mientras que la inflación promedio está dentro de lo esperado. En el ámbito fiscal, las medidas dirigidas a la consolidación fiscal en América Latina han dado lugar a una reducción del déficit primario, que pasaría de un promedio de 0,8\% del PIB en 2017 a 0,5\% del PIB en 2018.
} 


\section{REFERENCIAS}

1. Etienne CF. Investing in universal health in the Americas. Rev Panam Salud Publica. 2018;42:e90. https://doi.org/10.26633/ RPSP.2018.90

2. Cid Pedraza C, Matus-López M, Báscolo E. Espacio fiscal para salud en las Américas: ¿es suficiente el crecimiento económico? Rev Panam Salud Publica. 2018;42:e86. https://doi.org/10.26633/RPSP.2018.86

3. Matus-López M, Prieto Toledo L, Cid Pedraza C. Evaluación del espacio fiscal para la salud en Perú. Rev Panam Salud Publica. 2016;40(1):64-9.

4. Matus-López M, Cansino Pozo D, Cid Pedraza C, Valdés Romero W. Evaluación del espacio fiscal para salud en Bolivia. Rev Panam Salud Publica. 2018;42:e4. https://doi.org/10.26633/RPSP.2018.4

5. Prieto Toledo L, Montañez Ginocchio V, Cid-Pedraza C. Espacio fiscal para salud en Honduras. Rev Panam Salud Publica. 2018;42:e8. https://doi.org/10.26633/ RPSP.2018.8

6. Organización Panamericana de la Salud/ Organización Mundial de la Salud (OPS/ OMS). Espacio Fiscal para la Salud en Suriname. En prensa.

7. Rathe M, Hernández P, Van Mosseveld C, Pescetto C, Van de Maele N. Health accounts from past to present for a political arithmetic. Rev Panam Salud Publica. 2018;42:e89. https://doi.org/10.26633/ RPSP.2018.89

8. Petrera Pavone M, Jiménez Sánchez E. Determinantes del gasto de bolsillo en salud de la población pobre atendida en servicios de salud púbicos en Perú, 2010-2014. Rev Panam Salud Publica. 2018;42:e20. https://doi.org/10.26633/RPSP.2018.20

9. La Foucade A, Gabriel S, Scott E, Metivier C, Theodore K, Cumberbatch A, et al. Increased taxation on cigarettes in Grenada: potential effects on consumption and revenue. Rev Panam Salud Publica. 2018;42:e195. https:/ / doi.org/10.26633/RPSP.2018.195

10. Organización Panamericana de la Salud/ Organización Mundial de la Salud (OPS/ OMS). Espacio Fiscal para la Salud en América Latina. Serie Salud Universal, 2018. Washington D.C.: OPS, 2018.

11. Organización Panamericana de la Salud (OPS). Resolución CD53.R14. Estrategia para el acceso universal a la salud y la cobertura universal de salud. $53^{\circ}$ Consejo Directivo, $66^{\circ}$ Sesión del comité Regional de la Organización Mundial de la Salud para las Américas, 29 de septiembre al 3 de octubre de 2014. Washington, D.C.: OPS/OMS; 2014

12. Heller, P. Understanding fiscal space. Washington, D.C.: International Monetary Fund (IMF); 2005. Policy Discussion Paper, Issue $\mathrm{PDP} / 05 / 4$

13. Comisión Económica para América Latina y el Caribe (CEPAL), Panorama Fiscal de América Latina y el Caribe, 2018 (LC/ PUB.2018/4-P). Santiago: CEPAL; 2018.

14. Yates, R. Universal Health Coverage Series. Progressive taxes are key. Lancet. 2015;386(9990):227-9.

15. ReevesA, GourtsoyannisY, BasuS,McCoy D, McKee M, Stuckler D. Financing universal health coverage-effects of alternative tax structures on public health systems: cross-national modelling in 89 low-income and middle-income countries. Lancet. 2015;386(9990):274-80.

16. Swan T. Economic growth and capital accumulation. Econ Rec. 1956;32(2):334-61.

17. Becker G. Human capital. Nueva York: Columbia University Press; 1964.

18. Schultz T. Investment in human capital. Am Econ Rev. 1961;51(1):1-17.

19. Barro R. Determinants of economic growth. Massachusetts: The MIT Press; 1997.

20. Kalemli-Ozcan S, Ryder H, Weil D. Mortality decline, human capital investment, and economic growth. J Dev Econ. 2000;62(1):1-23.

21. Mayer D. Long-term impact of health on economic growth in Latin America. World Dev. 2001;29(6):1025-33.

22. Bloom D, Canning D, Sevilla J. The effect of health on economic growth: theory and evidence. NBER. 2001; 8587.

23. Howitt P. Health, human capital and economic growth: a schumpeterian perspective. Washington D.C.: Pan American Health Organization; 2005.

24. Jamison D, Lau L, Wang J. Health's contribution to economic growth in an environment of partially endogenous technical progress. En: G. López-Casanovas, B. Rivera \& L. Currais (eds.), Health and economic growth: Findings and policy implications, 67-91. Massachusetts: The MIT Press; 2005.

25. López-Casanovas G, Rivera B, Currais L. Introduction: The role health plays on economic growth. En: G. López-Casanovas, B. Rivera \& L. Currais, (eds.), Health and economic growth: Findings and policy implications, 1-16. Massachusetts: The MIT Press; 2005.

26. Van Zon A, Muysken J. Health as a principal determinant of economic growth. En G. López-Casanovas, B. Rivera \& L. Currais, (eds.), Health and economic growth: Findings and policy implications, 41-65. Massachusetts: The MIT Press; 2005.

27. Weil D. Accounting the effect of health on economic growth. Q J Econ. 2007;122(3): 1265-1306.

28. Osang T, Sarkar J. Endogenous mortality, human capital and economic growth. J Macroecon. 2008;30:1423-45.

29. Gupta M, Barman T. Health, infrastructure, environment and endogenous growth. J Macroecon. 2010;32:657-73.

30. Acemoglu D, Johnson S. Disease and development: the effect of life expectancy on economic growth. J Polit Econ. 2007;115(6): 925-85.

31. Alleyne G, Cohen D. Health, economic growth and poverty reduction. Report Working Group Commission on Macroeconomics and Health, 2002.

32. Sala-i-Martín X. Unhealthy people are poor people... and vice versa. Health and Economic Growth, MIT Press, 2005.

33. Wagstaff A. Child health on a dollar a day. Some tentative comparisons. Soc Sci Med. 2003;57:1529-38

34. Basu D, Das D, Basole A, Foley DK. The effect of public health expenditure on infant mortality: evidence from a panel of Indian States,
1983-84 to 2011-12. Economics Department Working Paper Series 199. Amherst: University of Massachusetts; 2015.

35. Organización Panamericana de la Salud/ Organización Mundial de la Salud (OPS/ OMS). Financiamiento de la Salud en las Américas. Salud en las Américas 2017. Washington, D.C.: OPS; 2017.

36. Organización Panamericana de la Salud/ Organización Mundial de la Salud (OPS/ OMS). Transformaciones de los sistemas de salud hacia la salud universal. Salud en las Américas 2017. Washington, D.C.: OPS; 2017.

37. Cashin C, Sparkes S, Bloom D. Earmarking for health: from theory to practice (No. WHO/HIS/HGF/HFWorkingPaper/17.5). Geneva: World Health Organization; 2017.

38. Cid C, Báscolo E, Morales C. La eficiencia en la agenda de la estrategia de acceso y cobertura universales en salud en las Américas. Salud Publica Mex. 2016;58(5): 496-503.

39. Báscolo E, Cid C, Pagano J P, Urrutia MS, Riego AD. El desafío de la sostenibilidad de los programas ampliados de inmunizaciones. Rev Panam Salud Publica. 2017;41:e160.

40. Moreno-Serra R, Smith P. Does progress towards universal health coverage improve population health? Lancet. 2012;380: 917-23.

41. Maruthappu M, Watkins J, Mohd Noor A, Williams C, Ali R, Sullivan R. Economic downturns, universal health coverage, and cancer mortality in high-income and middle-income countries, 1990-2010: a longitudinal analysis. Lancet. 2016;388: 684-95.

42. Maruthappu M, Ng KYB, Williams C, Atun R, Zeltner T. Government Health Care Spending and Child Mortality. Pediatrics. 2015;135(4).

43. Comisión Económica para América Latina (CEPAL). Estudio Económico de América Latina y el Caribe Evolución de la inversión en América Latina y el Caribe: hechos estilizados, determinantes y desafíos de política. Santiago: CEPAL; 2018.

44. Krugman P. End this depression now! Nueva York: W.W. Norton \& Company; 2012.

45. Thomson S, Figueras J, Evetovits T, Jowett M, Mladovsky P, Maresso A, et al. Economic crisis, health systems and health in Europe: impact and implications for policy. European Observatory. World Health Organization (Europe). McGraw Hill Open University Press; 2015.

46. Stuckler D, Reeves A, Loopstra R, Karanikolos M, McKee M. Austerity and health: the impact in the UK and Europe. European J Public Health. 2017;27(4):18-21.

47. Kwon H. Advocacy coalitions and health politics in Korea. Soc Policy Admin. 2007;41(2):148-61.

Manuscrito recibido el 15 de noviembre de 2018 . Aceptado para publicación el 6 de diciembre de 2018. 
ABSTRACT

\section{Fiscal space for sustainable financing of health systems and universal health}

Keywords Healthcare financing; health systems; taxes; health equity; efficiency; Americas.
The articles published in this series show that it is possible to create fiscal space for health in the countries. This requires specific decisions since economic growth is not enough to generate the additional resources needed. The studies analyze the benefit of reviewing the tax expenditures to identify exemptions - generally outdated and of low benefit for the countries -; arguments to increase taxes on products harmful to health; and credits and donations, which do not result in a viable source of income for governments. Fiscal efforts must be accompanied by an improvement in efficiency, and the progressive creation of new revenues is key to improving equity. It is necessary to improve the collection of fiscal resources.

To this end, it is necessary to develop a research and action agenda that understands the analysis of the fiscal space inserted in the processes of transformation and reform of health systems, including the technical aspects not yet addressed and studies of the social efficiency of the fiscal space for major development objectives such as the Sustainable Development Goals 2030. In the countries of the Region of the Americas it is essential to have efficient management to do more and better with more resources, even during adverse economic cycles. This should be evident at all levels, including solidarity for achieving better health outcomes, strategic purchase of health goods and services, and strengthening of budget planning systems.

RESUMO Os artigos publicados nesta série mostram que é possível criar espaço fiscal para a saúde nos países. Isso requer decisões específicas, já que o crescimento econômico não é suficiente para gerar os recursos adicionais necessários. Os estudos analisam o benefício de rever as despesas fiscais para identificar isenções-geralmente desatualizadas e de baixo benefício para os países-; argumentos para aumentar os impostos sobre os produtos prejudiciais à saúde; e créditos e doações, que não resultam em uma fonte viável de renda para os governos. Os esforços fiscais devem ser acompanhados por uma melhoria na eficiência, e a criação progressiva de novas receitas é fundamental para melhorar a equidade. É necessário melhorar a arrecadação de recursos fiscais.

Para tanto, é necessário desenvolver uma agenda de pesquisa e ação que compreenda a análise do espaço fiscal inserido nos processos de transformação e reforma dos sistemas de saúde, incluindo os aspectos técnicos ainda não abordados e estudos sobre a eficiência social do espaço fiscal para grandes objetivos de desenvolvimento, como os Objetivos de Desenvolvimento Sustentável 2030. Nos países da Região das Américas é essencial ter uma gestão eficiente para fazer mais e melhor com mais recursos, mesmo durante ciclos econômicos adversos. Isso deve ser evidente em todos os níveis, incluindo a solidariedade para alcançar melhores resultados de saúde, a compra estratégica de bens e serviços de saúde e o fortalecimento dos sistemas de planejamento orçamentário.

Palavras-chave Financiamento da assistência à saúde; sistemas de saúde; impostos; equidade em saúde; eficiência; Américas. 